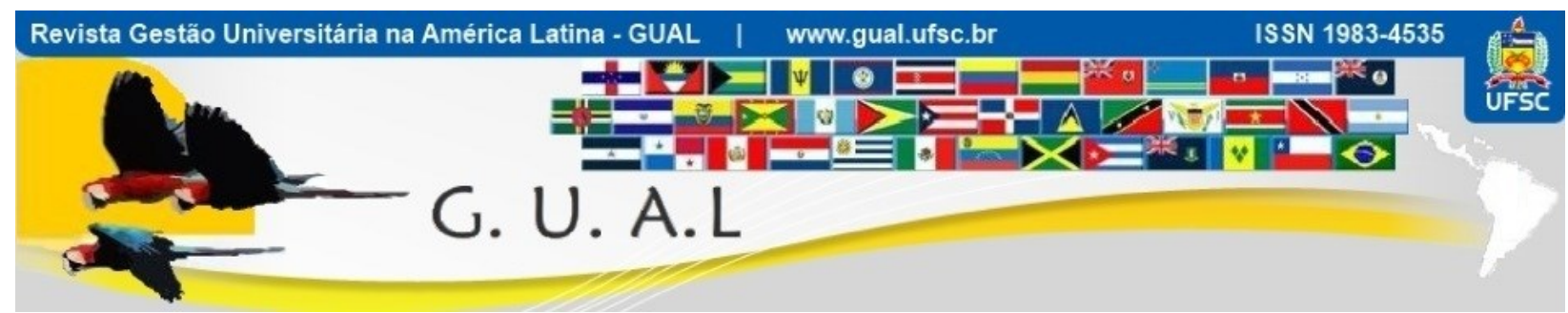

DOI: http://dx.doi.org/10.5007/1983-4535.2016v9n4p92

\title{
A ESCOLHA DE UMA UNIVERSIDADE VISTA SOB O PRISMA DA TEORIA DO COMPORTAMENTO DO CONSUMIDOR
}

\section{THE CHOICE OF A UNIVERSITY VIEWED FROM THE PERSPECTIVE OF THE THEORY OF CONSUMER BEHAVIOR}

Vera Lucia Telles Scaglione, Doutoranda Pontifícia Universidade Católica do Paraná - PUCPR vera.fpa@terra.com.br

Lizika Pitpar Goldchleger, Mestre Faculdade Cultura Inglesa - FCI lizika@,culturainglesasp.com.br

Recebido em 04/dezembro/2015

Aprovado em 14/outubro/2016

Sistema de Avaliação: Double Blind Review

Esta obra está sob uma Licença Creative Commons Atribuição-Uso. 


\title{
RESUMO
}

Este estudo tem como objetivo compreender o comportamento dos jovens na escolha de uma universidade, vista aqui, como uma relação de consumo, e mais especificamente como um processo de extensão da identidade. A pesquisa propõe-se a explorar os fatores concorrentes dessa escolha, passando por tópicos como: reputação e imagem organizacionais, considerando a influência dos rankings universitários, o comportamento do consumidor e a teoria da extensão do self através de posses. Foi realizado um estudo empírico com jovens, com uma abordagem metodológica qualitativa, por meio de entrevistas. Na etapa seguinte os dados foram categorizados e organizados através da análise de conteúdo. Os resultados obtidos evidenciam que, efetivamente, os jovens escolhem a universidade como uma relação de consumo e uma extensão da própria identidade.

Palavras-chave: Comportamento do consumidor. Escolha de universidade. Imagem organizacional. Rankings universitários. Reputação organizacional.

\begin{abstract}
The present study aims to understand the behavior of young people when choosing a university for their undergraduate studies. This process is understood here, as a consumer relationship, more specifically as an extension process of one's identity. The study, proposes to explore the competing factors involved in the youngsters'choice and involves such topics like organizational image and reputation. It also takes into account the influence of university rankings, consumer behavior and the theory of self-extension interpreted as possession. An empirical study has been conducted involving young people, and a qualitative methodological approach has been applied to interviews.In the following stage, data have been categorized and organized according to content analysis. The results point at the fact that young people choose a university just like an act of consumption and as an extension of their own identity.
\end{abstract}

Key words: Consumer behavior. Choice of a university. Organizational image. University rankings. Organizational reputation. 


\section{INTRODUÇÃO}

Nas últimas décadas tornou-se visível o crescimento da competitividade no segmento das Instituições de Educação Superior (IES) privadas. Dessa forma, o ingresso de novos alunos transformou-se num desafio para muitas IES, que até então não tinham essa questão como fundamental para sobrevivência financeira da instituição. Diante dessa realidade, surge a necessidade de uma gestão de Marketing dessas IES e a relação aluno-universidade passa a ser vista como consumo, e a compreensão do comportamento desse consumidor como extremamente necessária, o que justifica este estudo.

A escolha de uma universidade constitui-se num grande desafio para os jovens particularmente das classes $\mathrm{B}$ e $\mathrm{C}$, as quais, em função do cenário econômico atual, reúnem maiores possibilidades para realizar essa escolha. Neste estudo a classe A foi descartada uma vez que envolveria outros fatores específicos desse grupo. Este segmento social escolhe com outros critérios, entre eles apenas um grupo pequeno de Instituições de Ensino Superior tradicionais com excelente reputação.

O objetivo central deste estudo é compreender o comportamento dos jovens de classes $\mathrm{B}$ e $\mathrm{C}$ no que se refere à escolha de uma universidade e, principalmente, compreender os fatores que os influenciam esse momento. Reitere-se que esta opção leva em conta a relação aluno-universidade percebida como uma relação de consumo, podendo ser vista como um processo de extensão do Self se, especificamente, a escolha da universidade for analisada sob o prisma da Teoria do Consumidor.

Para atingir este objetivo, na primeira parte foi realizada uma revisão de literatura dos principais trabalhos encontrados sobre o tema. Em seguida, foram analisados aspectos que constituem a reputação, a imagem e a legitimidade de uma IES, considerada a questão atualmente tão em foco - da influência dos rankings universitários nesse processo. $\mathrm{Na}$ segunda parte, expõe-se a teoria da extensão do self através das posses, a partir do construto de Belk (1988); e por fim, a pesquisa de campo de tipo qualitativa, desenvolvida por meio de entrevistas com jovens nesse momento de escolha e pertencentes às classes sociais $\mathrm{B}$ e $\mathrm{C}$. Finaliza-se este trabalho analisando os resultados obtidos na coleta de dados à luz da teoria com algumas considerações e sugestão sobre o assunto. 


\section{FUNDAMENTAÇÃO TEÓRICA}

Na revisão de literatura foi possível perceber a existência de várias pesquisas similares sobre extensão da identidade a partir de posses; e que, praticamente todas, estavam direcionadas apenas a posses de objetos. No Brasil, diversos pesquisadores estudaram a relação entre posses de objetos e self estendido, sob diferentes enfoques, com destaque para: Castilhos et al (2006); Rossi et al (2006); Leão, Mello e Freitas (2008); Cavendon, Castilhos e Biasotto (2009); Bacha, Santos, Strehlau (2009). Já estudos de extensão do Self a partir de lugares, pessoas e partes do corpo, conforme preconizado por Belk (1988), tais como realizado neste trabalho, foram ainda pouco explorados.

Quanto à escolha de IES, do ponto de vista de Marketing, pela relação de consumo, apenas alguns trabalhos foram encontrados, entre os quais os de: Kotler e Fox (1994); Galli (2000); Alfinitto (2002); Dias (2005); Andaku (2006); Scadura (2008); Zavarrone e Romenti (2010) e Matos e Baptista (2011). Esses estudos questionam os fatores que influenciam os estudantes no momento da decisão por uma IES. A maioria chegou ao fator reputação da IES como o critério predominante nessa escolha, seguido de fatores que podem ser denominados de conveniência, como: valor das mensalidades, localização e infraestrutura das IES. A partir da revisão dessa literatura tomou-se o tema escolha e adotaram-se os critérios: reputação, imagem, legitimidade e, como fator preponderante nesse processo, a extensão do Self.

\subsection{REPUTAÇÃO, IMAGEM E LEGITIMIDADE}

A reputação institucional pode ser considerada como um fenômeno genuinamente construído pelo homem através da interação social e comunicação interpessoal (POWER et al, 2013). Para as autoras Zavarrone e Romenti (2010) a reputação é formada primeiramente a partir de percepções individuais que, a seguir, tornam-se opiniões compartilhadas e, por fim, transformam-se em consensos sociais, os quais formam a base da reputação de uma organização. Afirmam também que reputação é um conceito dinâmico que se forma juntamente com a história da organização, uma vez que se baseia na coerência e continuidade no tempo, de seus atributos e de seu comportamento.

A reputação representa uma imagem consolidada, uma opinião pública formada a respeito de algo e, diferentemente da imagem que, apesar de se tratar de uma construção mental, possui como características a inconstância e a volatilidade (LASBECK, 2007). 
A imagem é também uma construção social, porém, ao contrário da reputação formada pela percepção externa sobre a organização - a imagem é formada internamente e transmitida externamente. Assim sendo, será sempre positiva, uma vez que foi criada pela organização e tem, entre outros objetivos, o de melhorar a reputação organizacional (ROSSONI; GUARIDO FILHO, 2011). Para Kotler e Fox (1994, p.59) a “imagem é a soma de crenças, idéias e impressões que uma pessoa tem de um objeto".

Deephouse e Cartel (2005), a partir de um estudo realizado em organizações financeiras, comparam legitimidade e reputação, e ressaltam que ambas tratam-se de construções sociais, diferenciadas pelo fato de a legitimidade envolver a aceitação social, o respeito às normas, aos valores e as expectativas sociais, enquanto a reputação tem um caráter mais relacional, uma vez que envolve a comparação entre organizações.

Estudos trazem a ideia da relação positiva entre desempenho financeiro e reputação, e destacam que, quanto melhor o desempenho financeiro, melhor a reputação de uma organização (DEEPHOUSE; CARTEL, 2005; ROSSONI; GUARIDO FILHO, 2011). O tamanho da organização também influi positivamente: quanto maior o tamanho de uma organização, mais alta a sua reputação (GUARIDO FILHO, 2011).

A reputação universitária, assim como outros temas referentes à gestão universitária, é carente de literatura científica específica, visto que os modelos empresariais não se aplicam plenamente ao contexto universitário, o qual possui características bastante peculiares. A reputação universitária não possui uma definição particular, assim como sua mensuração é bastante difícil e questionável. Trata-se de um conceito bastante subjetivo, não vinculado apenas à qualidade acadêmica, à pesquisa e às boas práticas; mas também a um conceito multivariado, uma vez que pode ser percebido de diferentes formas pelos seus variados grupos de interesses: estudantes, familiares, professores, funcionários, e mercado em geral (ZAVARRONE; ROMENTI, 2010).

A classificação da reputação das organizações é feita através de rankings, que são sistemas de classificação que buscam simplificar, objetivar e racionalizar medidas de desempenho de organizações, ou seja, tentam criar uma escala hierárquica, a partir da qual determinado público pode conhecer a reputação de determinado segmento. 


\subsection{O SIGNIFICADO DOS RANKINGS UNIVERSITÁRIOS}

Mercados globalizados, como os atuais, são ambientes férteis para que prosperem rankings das mais variadas espécies, dentre eles os rankings universitários. Tais sistemas mostram-se importantes, pois possibilitam uma comparação simples e direta do desempenho educacional e da produtividade acadêmica de uma universidade, de uma nação - internamente e em comparação a outras. Muitas vezes os rankings universitários são entendidos como ferramentas para mensuração da qualidade e excelência do ensino superior e, podem também, ser considerados indicadores do desenvolvimento econômico e de vantagem competitiva de um país. Essas comparações são importantes para definição do status e reputação profissional dos próprios acadêmicos. E, por fim, úteis principalmente para o público em geral, que as considera no momento de escolha de uma universidade e de um curso, principalmente de pósgraduação. Vale destacar que esses rankings foram inicialmente criados para esse fim, depois ganharam outras finalidades (HAZELKORN, 2013).

Por essas razões, os rankings universitários têm atraindo cada vez mais espaço nos meios de comunicação social e ganham força e prestígio, comprovados pelo crescente espaço nas mídias. Atualmente existem diversos rankings universitários, alguns de prestígio mundial e outros regionais. A seguir, o Quadro 1 apresenta os dez principais rankings mundiais.

- Academic Ranking of World Universities (ARWU) (Shanghai Jiao Tong University), 2003

- Webometrics (Spanish National Research Council), 2003

- World University Ranking (Times Higher Education/Quacquarelli Symonds), 2004-09

- Performance Ranking of Scientific Papers for Research Universities (HEEACT), 2007

- Leiden Ranking (Centre for Science \& Technology Studies, University of Leiden), 2008

- World's Best Colleges and Universities (US News and World Report), 2008

- SCImage Institutional Rankings, 2009

- Global University Rankings (RatER) (Rating of Educational Resources, Russia), 2009

- Top University Rankings (Quacquarelli Symonds), 2010

- World University Ranking (Times Higher Education/Thomson Reuters-THE-TR), 2010

- U-Multirank (European Commission), 2011.

Quadro 1 Os Principais Rankings Universitários.

Fonte: HAZELKORN (2013).

No Brasil existem rankings regionais bastante conhecidos como o Guia do Estudante, publicado anualmente pela Editora Abril. O mais utilizado, na prática, apresenta os resultados extraídos das avaliações institucionais realizadas pelo governo, como o "Conceito ENADE", indicador que avalia o desempenho dos estudantes através do Exame Nacional de 
Desempenho de Estudantes, realizado pelo Instituto Nacional de Estudos e. Pesquisas Educacionais Anísio Teixeira (Inep).

Uma das principais críticas feitas à utilização dos rankings diz respeito à diversidade de indicadores utilizados em cada um deles. Também se deve levar em consideração a dificuldade de mensuração deles. Avaliar uma universidade é uma tarefa bastante difícil dada a complexidade de suas estruturas, implicações sociais, filosóficas, políticas, éticas, culturais e ideológicas existentes. Outro fator a ser considerado é a variedade de contextos, objetivos e missões de cada uma das universidades (DIAS SOBRINHO, 2000).

A visibilidade dos rankings tem sido tão ampla que até mesmo figurar entre as últimas posições concede grande destaque à universidade, e gera um fortalecimento e valorização da marca junto ao seu público. Destacar-se nestes rankings é tão importante como imagem que algumas IES acabam selecionando alunos que se sobressaem nas provas e exames, como estratégia aproveitando-os em anúncios publicitários para melhorar seu desempenho e/ou imagem no mercado (HAZELKORN, 2013).

Halzelkorn (2013) apresenta uma correlação positiva entre a posição de uma IES no ranking e as escolhas feitas pelos alunos. Relata, também, que isso acontece pelo fato de os estudantes relacionarem essa posição das IES nos rankings à obtenção de vantagens pessoais, principalmente de ordem financeira.

\subsection{COMPORTAMENTO DO CONSUMIDOR - POSSES COMO EXTENSÃO DO SELF}

Entender o comportamento do consumidor, suas motivações e seus impulsos, sempre foi um dos grandes objetivos do marketing. Neste estudo adotou-se a teoria formulada por Belk (1988), que versa sobre as posses como forma de extensão da identidade.

Belk (1988) em seu artigo seminal: Possessions and the extended self, a partir de uma extensa revisão bibliográfica e algumas incursões pela teoria psicanalítica, defendeu a tese de que para entender o comportamento do consumidor é preciso compreender o sentido por ele atribuído às suas posses. Assim, buscou em seu estudo, através do exame da relação entre bens, posses e senso de self, entender como os consumidores usam suas posses para estender e fortalecer seu senso de self e delimitar quem eles realmente são. Self, para o autor, significa a representação que o indivíduo faz de si mesmo, como um sinônimo de identidade e, no caso do self estendido, estaria incluída a soma de suas posses. 
Posses, nesse caso, não são apenas referentes a objetos, mas também representam algo bem mais amplo, como: lugares, pessoas, partes do corpo, animais, entre outros. Belk (1988 p. 139) conceitua posses da seguinte forma: "se definimos posses como as coisas que chamamos de nossas, estamos dizendo que somos a soma de nossas posses". Torna-se imperioso assinalar que Belk (1988) não se refere a posses simples, e sim àquelas revestidas de energia afetiva, ou seja, seriam as posses que se revestem de valor simbólico para o indivíduo.

Para Belk (1988) a essência do ser está contida na inseparável tríade do: ter, fazer e ser. O autor chegou a essa definição a partir de releituras de Sartre (1943, apud BELK, 1988), que coloca o ter e o ser como pontos centrais da existência humana; e de Marx (1967, apud BELK, 1988), para quem a essência da existência humana estaria no fazer, ou seja, no trabalho. Dessa essência, Belk (1988) extraiu duas premissas:

a) A relação ter-fazer-ser é de grande importância para a compreensão do comportamento de consumo;

b) O entendimento do self estendido ajuda na compreensão mais ampla do ser humano.

Belk (1988) encontrou evidências da assertividade de suas premissas sobre a extensão do self através de posses distintas como: roupas, casa, família, amigos, lugares, partes do corpo, residências, ideias e coleções. Para comprovar sua tese, o autor exemplifica com o sofrimento resultante em algumas situações nas quais ocorrem perdas não intencionais de bens. Nesses casos, os indivíduos relatam essas ocorrências como momentos de luto e depressão, em que experimentam estes sentimentos como perda de uma parte de si mesmo.

Para melhor respaldar suas premissas recorre a uma breve incursão na Teoria Psicanalítica, especialmente o que postula Winnicott (1953) sobre a formação da identidade. Winnicott propõe uma teoria do desenvolvimento precoce da identidade, do self e das emoções a partir do vínculo materno, por ele denominado de holding, visto como relação objetal. De acordo com a Winnicott (1953), a relação mãe-bebê está na base de todas as outras relações que se desenvolverão a partir dela, e a qualidade desse vínculo determina a constituição da identidade e consequentemente influencia todos os relacionamentos afetivos futuros.

Belk (1988) recorre a essa teoria de Winnicott uma vez que ela trabalha a diferenciação eu-mundo. De acordo com Winnicott (1953), num primeiro momento o bebê não tem capacidade de perceber a diferença entre ele e o outro, ou entre o eu e os objetos; essa 
separação ocorrerá gradativamente apenas num estágio bem mais desenvolvido, com o avançar adiante da idade da criança. Belk (1988) postula que muitas vezes os objetos, ao longo da vida, passam a fazer parte do eu, num processo inverso ao proposto por Winnicott (1953): novamente o eu funde-se aos objetos.

Belk (1988) ainda buscando apoio na Psicanálise; cita Klein (1935, apud BELK, 1988) para quem a relação objetal entre pessoas e objetos nunca é simples, pois sempre há outras pessoas em disputa pelo mesmo objeto.

\section{PROCEDIMENTOS METODOLÓGICOS}

A pesquisa realizada pode ser classificada como qualitativa. As abordagens qualitativas são particularmente indicadas quando o objetivo é interpretar fenômenos de consumo (AYROSA; SAUERBRONN, 2004). Quanto aos fins, a pesquisa tem caráter exploratório-descritivo. Exploratório pelo fato de o comportamento do consumidor ser ainda relativamente novo e de haver poucos estudos que abordem a questão da escolha de uma universidade pela a ótica do consumo; e descritivo porque visa descrever as percepções, os desejos e motivações desse consumidor, especificamente.

A pesquisa de campo foi realizada por meio de um roteiro semi-estruturado. No que se refere ao tamanho da amostra, segundo McCraken (1988), é mais importante trabalhar profundamente com poucos indivíduos do que superficialmente com um número grande de pessoas. Assim, torna-se possível realizar uma análise aprofundada dos dados obtidos.

O universo dessa pesquisa são estudantes, na iminência de escolher uma Instituição de Educação Superior, ou que o fizeram há menos de dois anos. Os dados empíricos foram coletados com 23 jovens, com idades entre 16 e 24 anos, classes B e C.

A fase de coleta dos dados compreendeu os meses de maio e junho de 2015. Apenas uma entrevista foi descartada devido ao fato do respondente não se enquadrar nas classes sociais eleitas para a pesquisa. A seguir, o Quadro 2 apresenta o perfil dos entrevistados e a respectiva codificação adotada para a análise dos dados. 


\begin{tabular}{|c|c|c|c|}
\hline $\begin{array}{c}\text { CÓDIGO DOS } \\
\text { RESPONDENTES }\end{array}$ & IDADE & ESCOLARIDADE & $\begin{array}{l}\text { TRABALHA? } \\
\text { S OU N }\end{array}$ \\
\hline 1 & 18 & $1^{\circ}$ ano faculdade & S (Estágio) \\
\hline 2 & 17 & $3^{\circ}$ ano do E. Médio & $\mathrm{N}$ \\
\hline 3 & 16 & $3^{\circ}$ ano do E. Médio & $\mathrm{N}$ \\
\hline 4 & 17 & $3^{\circ}$ ano do E. Médio & $\mathrm{N}$ \\
\hline 5 & 17 & $3^{\circ}$ ano do E. Médio & $\mathrm{N}$ \\
\hline 6 & 20 & $1^{\circ}$ ano faculdade & S (Estágio) \\
\hline 7 & 18 & E.Médio completo & $\mathrm{S}$ \\
\hline 8 & 19 & $3^{\circ}$ semestre faculdade & $\mathrm{S}$ \\
\hline 9 & 18 & $1^{\circ}$ ano de faculdade & $\mathrm{S}$ \\
\hline 10 & 22 & $1^{\circ}$ ano de faculdade & $\mathrm{N}$ \\
\hline 11 & 19 & $2^{\circ}$ ano de faculdade & S (Estágio) \\
\hline 12 & 20 & $1^{\circ}$ ano de faculdade & $\mathrm{S}$ \\
\hline 13 & 23 & $1^{\circ}$ ano de faculdade & $\mathrm{S}$ \\
\hline 14 & 21 & $1^{\circ}$ ano de faculdade & $\mathrm{N}$ \\
\hline 15 & 23 & $\begin{array}{l}\text { Sup. Compl/ } 1^{\circ} \text { ano de } \\
\text { faculdade }\end{array}$ & $\mathrm{S}$ \\
\hline 16 & 20 & $1^{\circ}$ ano de faculdade & $\mathrm{N}$ \\
\hline 17 & 20 & E.Médio Completo & $\mathrm{S}$ \\
\hline 18 & 24 & $1^{\circ}$ ano de faculdade & $\mathrm{S}$ \\
\hline 19 & 17 & $1^{\circ}$ ano de faculdade & $\mathrm{S}$ \\
\hline 20 & 20 & $1^{\circ}$ ano de faculdade & $\mathrm{S}$ \\
\hline 21 & 24 & Sup. Completo & $\mathrm{N}$ \\
\hline 22 & 18 & $1^{\circ}$ sem. Faculdade & S (estágio) \\
\hline
\end{tabular}

Quadro 2 Perfil e Codificação dos respondentes.

Fonte: Elaborado pela autora a partir dos dados obtidos nas entrevistas.

Para o tratamento dos dados coletados foi utilizada a técnica da análise de conteúdo, conforme Bardin (1977), que se caracteriza como uma técnica de interpretação dos dados, que busca compreender o que está sendo dito a respeito de algo, do conteúdo manifesto na comunicação. Assim, a partir da fala dos entrevistados, constituíram-se as categorias de análise eleitas para atingir o objetivo proposto nesta pesquisa. Para tanto, foram criadas as categorias analíticas expectativas e qualidade do serviço ofertado; características para aquisição do serviço: conveniência e comodidades; reputação, imagem institucional e Rankings; e extensão do Self.

\subsection{APRESENTAÇÃO E ANÁLISE DOS DADOS}

A seguir serão apresentados os dados levantados nas entrevistas e comentados dentro de suas respectivas categorias de análise. 


\subsubsection{Expectativas e qualidade do serviço ofertado}

Uma vez que se pretende compreender o comportamento no que se refere à relação de consumo, entende-se que tudo comece com a busca de informações sobre o serviço que se pretende adquirir, no caso um curso superior em uma IES. A maioria dos jovens entrevistados apontou a utilização da internet para esse fim, principalmente a visita aos sites das IES (32\%). Os professores são apontados como a segunda maior fonte de informações. As falas de alguns respondentes ilustram os casos dos que utilizam outras fontes de informação ou complementam as informações encontradas na internet.

R3: "...com os professores da escola e do cursinho. Eles conhecem bem as faculdades."

R4: "Consigo referências com a família."

R5: "Visitei IES. Olhei matrizes e greves."

R11: " Troca de informações com professores. Não costumo visitar sites."

Percebe-se em grande parte dos respondentes que já fizeram uma escolha, que não houve um aprofundamento maior na busca ou confronto de informações. Poucos recorreram a visitas às IES e conversas com os coordenadores dos respectivos cursos e com alunos e exalunos do local escolhido. Pode-se sugerir que a questão das informações, um dos pontos racionais dessa decisão, não é considerada tão importante como seria de se esperar.

\subsubsection{Características para aquisição do serviço: conveniência e comodidade}

No que se refere às características para aquisição do serviço, ou seja, as vantagens, interesses e a conveniência na escolha, essa categoria configurou como a que gera maior atenção e preocupação para esses jovens. Interessam dados bastante objetivos como gratuidade ou valor das mensalidades, localização da IES, horário em que o curso é ofertado, se integral ou parcial, grau de dificuldade para ingresso e oferta de possibilidade de intercâmbios no exterior. Esses são os indicadores usados para a tomada final da decisão, o que revela similaridade com outras relações de consumo. Sobre a gratuidade das IES, identificou-se:

R1: "Sim. Meu pai preferia as públicas."

R2: "Muito. Não tem como pagar. É fundamental."

R3: "Sim. Medicina tem um preço muito elevado. São 5,6 mil reais."

R15: "É um fator importante pra saber se posso pagar." 
Também emergiram falas que expressam a preocupação com o ingresso nas IES escolhidas em relação aos vestibulares.

R1: "Preocupava - Fiz cursinho."

R2: " Sim. Mas não deixaria de escolher pela dificuldade."

R3: "Preocupa. Muita concorrência por média, você tem que gabaritar! Quase não têm vagas para Medicina."

No que se refere à oferta de intercâmbio, os jovens não se mostraram muito preocupados com esse. Como é o caso dos comentários dos respondentes R3 e R4:

R3: "Não preocupa, todas as públicas tem o Ciências sem Fronteiras".

R4: "...mais ou menos, isso eu posso resolver sozinho."

Outras pesquisas realizadas sobre o tema escolha profissional, como as de Galli (2000); Alfinitto (2002); Dias (2005); Andaku (2006); Scadura (2008), também chegaram a resultados similares sobre a importância das conveniências ou comodidades na decisão sobre a escolha de uma IES.

\subsubsection{Reputação, imagem institucional e Rankings}

A reputação e a imagem institucional, aliadas diretamente à questão da qualidade do serviço ofertado, apresentam-se como representativas e como preocupação unânime entre os entrevistados. Já, quanto ao fato de ser o melhor curso, as opiniões do grupo se dividiram: alguns acham relevante, mas não determinante, e que não necessariamente precisa ser o melhor curso, ou a melhor IES, mas uma das melhores. Parecem ter claro que somente a imagem e a reputação não resolvem, de maneira que apontam a importância do protagonismo do próprio aluno, como fundamental para o êxito nessa etapa, conforme as explanações.

R1: " Quem faz a faculdade é o aluno."

R4: " Qualidade tem que estar presente. "

R6: " Sim. Quem faz a faculdade é o aluno, não pode ficar parado!"

R12: " É bom quando ela é a melhor, mas se ela for uma das melhores já basta."

R20: " Sim. Porém só nome não garante qualidade."

A utilização e acompanhamento dos Rankings, assim como dos dados do ENADE, para fins da escolha profissional, não consideram nem um ponto primordial e nem aparentam ser confiáveis, conforme expresso nas palavras dos estudantes: 
R12: "Não acompanho, mas acho importante para saber da qualidade dos cursos ofertados." R14: "Dão um panorama da estrutura da faculdade com um todo." R16: "Acho-os relevante, mas não de 100\% confiança!"

A maioria dos estudantes desconhece os conceitos sobre as IES e não acompanha o ranking das universidades

Nesse ponto é possível perceber que o fato de não acompanharem os resultados das classificações das instituições os estudantes deixam de aproveitar uma importante fonte de informações sobre as IES. Os dados encontrados não corroboram com os de Halzelkorn (2013) que encontrou uma correlação positiva entre a posição de uma IES no ranking e as escolhas feitas pelos alunos.

\subsubsection{Extensão do Self}

Extensão do Self compreendida no sentido postulado por Belk (1988), que inclui a tríade do ser, ter e fazer e, consequentemente, o entendimento de que as posses fariam parte do ser. A extensão do Self representa o foco deste estudo.

Questões sobre o quanto entende a IES que frequentam ou passarão a frequentar, como uma continuidade do "eu" também foram verificadas. No que diz respeito à obtenção de status social a partir do curso ou da IES que frequentam ou frequentarão, a maioria respondeu prontamente que sim (64\%), o que corrobora com o pressuposto central deste estudo: a IES aparecendo como extensão do Self, conforme pode ser apreciado nas falas abaixo.

R3: "Com certeza confere, principalmente no futuro..."

R11: "Confere até além da minha realidade. A faculdade tem um tom de elite."

R21: "Sempre busquei estar entre os melhores..." "...agora após seis meses, nem percebo mais as minhas diferenças..."

Porém, pode-se perceber como subjetivo esse sentimento de "pertencimento" e de "ganho de status social", uma vez que - quando perguntados sobre a importância do status dos demais alunos - disseram ser irrelevante. Logo, é possível aferir que se trata mais de uma percepção de um status geral que real ou pessoal.

Quanto à importância da opinião do grupo social sobre sua escolha, demonstraram não se preocupar com esse ponto e que tal escolha já estaria tão arraigada ao seu "eu", que seria praticamente impossível persuadi-los, uma vez definida a opção. Ainda assim, preocupam-se mais com a opinião da família, que com a dos demais, conforme externado nestas manifestações: 
R2: "Só se trouxer argumentos racionais".

R7: "Apoiaram. Mesmo que não permanecesse com a escolha.

R12: "Apenas com o que os meus pais pensam."

R22: "Falavam para terminar o curso Técnico. Primeiro. Não acreditavam, davam muitos palpites. Aprendi a ignorar."

Manifestações semelhantes foram recorrentes nas demais entrevistas, o que confirma a “extensão do Self", com a incorporação da escolha como posse e o "eu" inseparável do objeto e evoca a ideia de relação de consumo. Conforme Belk (1988), tal confirmação do entendimento da escolha de uma IES como uma relação de consumo, auxiliará no caminho para a ampliação do conhecimento do comportamento desse consumidor e consequentemente de como realiza a escolha de uma IES.

\section{CONSIDERAÇÕES FINAIS}

Este estudo tem como objetivo compreender o comportamento dos jovens na escolha de uma Instituição de Educação Superior, entendida como uma relação de consumo. Visa também verificar até que ponto essa opção pode ser entendida, pela óptica da Teoria do Comportamento do Consumidor, como um processo de extensão da identidade do construto de Belk (1988). Nesse sentido, constatou-se que a escolha de uma universidade acontece realmente como uma relação de consumo, e a universidade pode ser vista como uma posse, ou seja, como uma extensão do Self.

Tal comportamento de consumo, conforme os dados da pesquisa de campo com jovens das classes sociais $\mathrm{B}$ e $\mathrm{C}$, leva à valorização excessiva de questões relacionadas a aspectos como a conveniência e comodidade. Dessa forma, o "custo" do serviço, torna-se uma prioridade, exatamente como ocorre em outras situações normais de consumo. Fatores apontados como fundamentais foram: localização e horário de oferta. Estudos anteriores realizados sobre o tema no Brasil, como o de Galli (2000); Alfinitto (2002); Dias (2005); Andaku (2006); Scadura (2008), também destacam a importância atribuída a fatores ligados à conveniência.

Por outro lado, a preocupação com o aprofundamento e verificação de informações relevantes, não são prioritários. A Internet mostra-se atualmente como o canal mais utilizado, seguida pelas informações transmitidas pelos professores. As visitas às universidades configuram somente no terceiro lugar de importância. 
A reputação universitária revelou-se construída socialmente através das interações entre as pessoas, formada primeiramente em nível individual e, depois, compartilhada (ZAVARRONE; ROMENTI, 2010). A percepção da reputação é variável quando observados os diferentes grupos sociais. Aparece bastante associada à qualidade, fator considerado fundamental por todos os entrevistados. Não foi explicitada a preocupação específica com o fato de o curso ou a IES escolhida serem considerados os melhores, importa que garantam um nível aceitável de qualidade. Os jovens têm muito clara a consciência de que são protagonistas dessa história e, como tal, responsáveis também pelo produto final.

Apesar de os Rankings representarem uma forma segura e fácil de comparação, e serem conhecidos pelos jovens, ainda assim não são aproveitados como indicadores para respaldar suas escolhas, pois não conseguem perceber o verdadeiro potencial informacional desses instrumentos.

Como outras pesquisas que procuram entender o comportamento do consumidor, e especificamente as posses como extensões da própria identidade; este estudo procurou elucidar até que ponto as universidades escolhidas passam, como outros tipos de posses a ser consideradas como uma extensão da identidade. Quase unânime dentre os jovens, emergiu a ideia de obtenção e/ou mudança de status social, a partir da escolha de uma Instituição de Ensino Superior, o que evidencia a expectativa que a universidade será uma extensão do próprio eu.

Como forma de enriquecimento desse debate sugere-se uma agenda para estudos futuros que busquem compreender esse comportamento em outros extratos sociais e, como contribuição espera-se colaborar com a gestão de marketing das universidades.

\section{REFERÊNCIAS}

ALFINITO, S. Determinação de atributos de preferência do consumidor na escolha de uma instituição de ensino superior no Distrito Federal. Brasília: UCB (Dissertação de Mestrado), 2002.

AYROSA, E. A. T.; SAUERBRONN, J. F. R. Uma introdução ao uso de métodos qualitativos de pesquisa em comportamento do consumidor. In: VIEIRA, Marcelo M. F.; ZOUAIN, Deborah M. Pesquisa Qualitativa em Administração. Rio de Janeiro: Editora FGV, 2004.

BACHA, M. L., SANTOS, J.; STREHLAU, V. Renda Baixa: O Self estendido na posse do celular. In: XII Semead 2009 Empreendedorismo e Inovação, São Paulo, v.1. p. 1 16, 2009. 
BELK, R. W. Possessions and the extended self. Journal of Consumer Research, v. 15, n.2, p. 139-168, Sep., 1988.

CAVENDON, N. R.; CASTILHOS, R.; BIASOTTO, L. D. Consumo, colecionismo e identidade dos bibliófilos: uma etnografia em dois sebos de Porto Alegre. Disponível em: www.scielo.br/pdf/ha/v13n28/a14v1328.pdf. Acessado em: 10 de janeiro de 2014.

CASTILHOS, R. et al, Self Estendido e Posse no Local de Trabalho: Uma Réplica no Contexto Brasileiro. Anais do II EMA. ANPAD, 2006.

DIAS, C. C. Os fatores de influência no processo decisório do aluno universitário. Projeto de Pesquisa, Centro Universitário Vila Velha, Espírito Santo, 2005.

DIAS SOBRINHO, J. Qualidade, Avaliação: do SINAES a Índices. Avaliação, v.13, n. 3. p. 817-825, 2008.

HAZELKORN, E. How Rankings are Reshaping Higher Education in Climent, V., Michavila, F. and Ripolles, M. (eds): Los Rankings Univeritarios: Mitos y Realidades, Ed. Tecnos, 2013.

FOX, K. F.; KOTLER, P. Marketing Estratégico para Instituições Educacionais. São Paulo: Atlas, 1994.

GALLI, G. Atributos Determinantes na Escolha de Instituições de Ensino Superior na Região do Vale do Rio dos Sinos. 2000. 94 f. Dissertação/Mestrado em Administração e Negócios, Pontifícia Universidade Católica do Rio Grande do Sul, 2000.

LASBECK, L. C. A. Imagem e reputação na gestão da identidade organizacional. Organicom, v. 4, n. 7; 2007. Disponível em:

http://revistaorganicom.org.br/sistema/index.php/organicom/article/viewFile/112/131. Acesso em 05 jan. 2014.

LEÃO, A. L. MELL O, S. C.; FREITAS, G. K. O alinhamento do "eu" (footing) em interações sociais por meio dos recursos simbólicos das marcas, In: III EMA ENNPAD, Anais III EMA, Enanpad , 2008

MATOS S.; BAPTISTA, P. Grupos de Referência como Fator de Influência na Escolha de uma Instituição de Ensino Superior. VIII Simpósio de Excelência e Gestão em Tecnologia. SEGET, 19 a 21 de out., Resende: 2011

McCRACKEN, G. The long interview. Newburry Park: Sage Publications. 1988.

POWER M. et al. Reputational Risk as a Logic of Organizing in Late Modernity. Organization Studies 30, Sage Publications, p. 301-324, London, 2009.

ROSSONIE. R. ; GUARIDO FILHO L. O que faz um nome? Antecedentes da Reputação das Empresas de Capital Aberto Brasileiras. XXXV Encontro da ANPAD, Rio de Janeiro, 4 a 7 de set. de 2011. 
SCARDUA, G. S. M. Critérios utilizados na escolha de instituições de ensino superior privadas de Salvador. Dissertação apresentada ao Programa de pós-graduação em Administração Mestrado Profissional, Faculdade de Administração, Universidade Federal da Bahia, 2008.

WINICOTT. D.W. Transitional Objects and Transitional Phenomena, International Journal of Psychoanalysis, $n^{0} 34$ vol. 2, p. 89-97, 1953.

ZAVARRONE E.; ROMENTI S. RePAC Um modelo per la mensurazione della reputazione per l'Academia. Sis-Magazine - Online Magazine della Società Italiana di Statistica, Itália, 2010. 